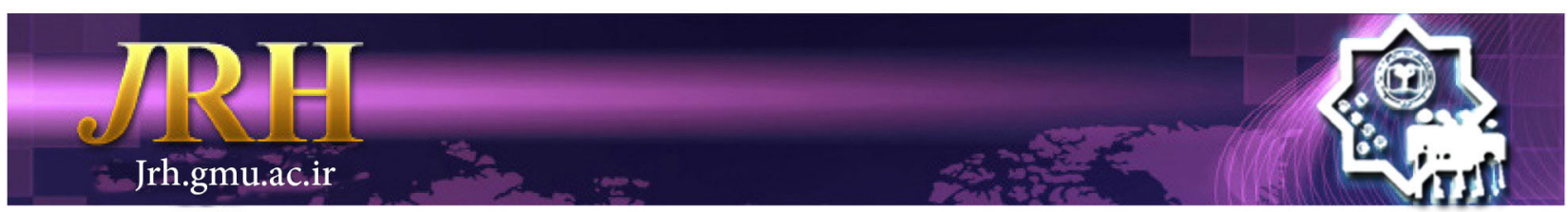

\title{
Awareness and observation of patients' rights from the perspective of patients and nurses
}

Fatemeh Barati ${ }^{1}$, Alireza Atarodi ${ }^{2}$, Bibi Aghdas Asghari ${ }^{1}$, Ahmadreza Atarodi $^{3}$

\author{
Journal of Research \& Health \\ Social Development \& Health Promotion \\ Research Center \\ Vol. 9, No.4, Jul \& Agu 2019 \\ Pages: $315-323$ \\ DOI: $10.29252 / j r h .9 .4 .315$ \\ Original Article
}

1. Department of Social Sciences, School of Humanities, Islamic Azad University Gonabad Branch, Gonabad, Iran

2. Correspondence to: Department of Medical Knowledge and Information Science, Paramedical College and Social Development \& Health Promotion Research Center, Gonabad University of Medical Sciences, Gonabad, Iran

Email: aratarodi1387@yahoo.com

3. Department of Medicine, Student Research Committee, Gonabad University of Medical Sciences, Gonabad, Iran

Received: 27 Oct 2018

Accepted: 9 Apr 2019

How to cite this article: Barati F, Atarodi A, Asghari BA, Atarodi A. Awareness and observation of patients' rights from the perspective of patients and nurses. J Research \& Health2019; 9(4): 315-323.

\begin{abstract}
People have some rights to use it for living, patients are people and they should be aware of their rights. The purpose of this study was to determine the level of awareness and observation of patients' rights and the factors affecting it from the perspective of the patients and nurses among all hospitalized patients and the nurses working at Bohlool hospital in Gonabad city. 100 patients and 100 nurses were selected as the statistical sample. Data were collected using the patients' rights questionnaire of bokai. The priority of the patients' rights were as the followings: respect to patient privacy and secrecy principle (96\% Moderate and Good), receiving desirable health services (92\% Moderate and Good), access to an efficient complaints system (90\% Moderate and Good), patient's access to appropriate and adequate information ( $91 \%$ Moderate and Good), and the right to making choice and decision freely for the patients (76\% Moderate and Good) in receiving hospital services, respectively. Also, there was a relationship between the level of awareness and observance of patients rights with demographic variables among the nurses. The results of the research showed that the lack of the patients' awareness from their health and treatment rights and the enforcement lack of patients' rights charter by the health and medical personnel can endanger health, life and safety of the patients. Therefore, the awareness and observance of these rights seemed necessary.
\end{abstract}

Keywords: Awareness, Hospital, Nurses, Patients' Rights

\section{Introduction}

Patient satisfaction is one ofeffectiveness criteria for hospital services. Patient satisfaction requiresobservation of patient rights. One of the most important principles of nursing is respect to human rights and patients' dignity. Davati argues that nurses are one of the main pillars of the patient rights claimers in hospitals [1]. Today, despite the efforts of physicians and staffs in the health sector and the availability of extensive facilities, the level of dissatisfaction and patient complaints has increased.

Sarbaz concluded in his study that the complications of medical procedures that lead to patients' complicant had a profound and serious impact on the patients' life and their families, a long and adverse effect on patients' occupation and social life and it even causes anxiety, stress, depression and isolation for the patients [2].

According to Al-Moujel, the major part 
of patients' satisfaction in health centers is observing their rights and helping them to meet their needs by staff. Health care recipients are demanding the observation and enforcement of the patient rights charter and expect their rights to be respected while meeting their health needs [3].

Joseph stated that the observance of the patients' rights charter would improve the relationships between the patient and health personnel. Patients' awarness of their rights increases the quality of care and reduces the costs. Another important issue is that awaring patients, sharing them in decision making and respecting to their rights will speed up their recovery, reduce hospital admissions and hinder from irreparable mental and physical harms [4].

The observance of the Patient Rights Charter improves the relationship between patient and health care staffs faster. Successful communication helps the complete implementation of medical orders by the patient, increase the care quality and significantly reduce the patient complaints [5].

Aydemir and colleagues quoted from Huton that:"Further efforts are needed to stabilize patient rights and strengthen their participation in their own health decision-making" [6]. According to Hooshmand, the rights of the patient can not be achieved only on the basis of ethical principles and some mechanisms should be provided by the formulation of rules and guidelines for monitoring and continuous controling these rights [7].

Rider and Makela conducted a survey in several countries, including Finland, Lithuania, Malaysia, South Africa and the United Kingdom and concluded that the patient rights in various countries were differently affected by economic, social, ethical and cultural conditions. The development and enforcement of laws protecting these rights are also affected by such conditions [8]. Various studies have shown that both the level of awareness and observation of patient rights is not in a good status. Houshmand et al. in their study concluded that the nurses knowledge about patient rights to receive essential information in the stages of diagnosis and progression disease is moderate [7].

The study of Arab and colleagues entitled "Managers' knowledge on patient rights in Tehran hospitals" shows that the knowledge level of managers at Tehran public hospitals about patient rights was respectively, 34.5\% good, $48.5 \%$ moderate and $17 \%$ weak. Also, the education, the field of study and the passing of management courses were three affecting factors onmanagers' knowledge about patient rights [9]. Paying attention to the patient rights and more important than it, observing these rights are essential and indisputable to all members of the medical team, including physicians and nurses. Patient satisfaction is one of the important indicators of effectiveness, efficiency and quality of health services [10]. In addition, due to the special importance of medical science in Islam, andtraining the specialists and commited physicians in universities, the respect and dignity of patients in educational hospitals should be observed [11]. Observing the information of the patient, respect to the patient's desire, fairness, and paying attention to the patient interests are among the important issues to be considered in clinical education. In order to observe the patient respect and rights, personnel must learn to keep the patient information confidential. The personnel are not allowed to conduct educational clinical examinations on patients without their informed consent.

In addition, increase in the educational efficiency and patient satisfaction is dependant to the patient knowledge about the personnel role. Different studies such as Khodamoradi et al., Larijani et al. and Vivan on the knowledge of different medical personnel show that the level of personnel knowledge in this regard is not appropriate [12-14]. Shiraz et al. showed that the knowledge about medical ethics among Internist medical personnel and surgical assistants in the surgical ward of Karachi hospitals was very weak [15]. In another study, some patients reported that they 
were treated as non-human, and they never felt that responsivenesswas a part of the education process [16].

Based on the results of the above studies, it seems that the awareness level of health personnel about patient rights is not appropriate and the cause of this issue is their student educational period. Because, the low level of knowledge may be due to inadequacy of the courses offered in their student period [1216]. Therefore, the purpose of this study was to determine the awareness and observation level of patient rights and its effective factors from the viewpoint of patients and nurses at a hospital.

\section{Method}

The statistical population included all employed nurses and patients admitted during the first three months of 2018 at Allameh Bohlool hospital in Gonabad city in north-eastern of Iran. With simple random sampling method, 100 patients and 100 nurses were selected as the statistical sample using Cochran's formula. Data collection was done using Bokai standard questionnaire. The validity of the questionnaire was confirmed by Bokai et al. and the content validity was assessed based on the review of the texts, articles and opinions of 10 professors. The reliability of this questionnaire was evaluated using a re-test and it also was previously used in other studies [18-17]. The criteria for entering the study included the following: to participate willingly in the study, the time of admission for at least 24 hours, the absence of clear mental disorder and the ability to cooperate. Exclusion criteria also included unwillingness to cooperate and not being able to respond questions for any reason.

Datacollectingtoolwasathree-partquestionnaire including demographic information (age, gender, education degree with options like: prediploma, diploma, postgraduate, bachelor, master degree and higher, and marital status for both patients and nurses, and also working period only for nurses. The second part of the questionnaire was related to the awareness level of patient rights charter ( 7 items). Besides, the third part of the questionnaire was related to the satisfaction degree for the observance of the patientrights charter (7 items), which addressed and measured different aspects of the patient rights charter regarding its awareness and observance.

The questionnaires were distrubuted to study samples to get completed. The method of rating scale of knowledge and observation on patient rights was provided in three options of good (score 2), moderate (score 1 ) and poor (zero score). The scores were graded from a minimum to a maximum (014) including seven questions for each item. Since there was no clear cut off point for this score, the researchers decided to divide the abovementioned range into three equal intervals: weak (0-4), medium (5-9), and good (10-14). After analyzing the data, the results were presented in the relevant tables. After obtaining permission from the research director of the Islamic Azad University of Gonabad, the research unit of Allameh Bohlool Gonabadi hospital, hospitalized patients and nurses working at Allameh Bohlool hospital, and with the adherence to the research ethics, such as informed consent, the principle of confidentiaity and the observation of patients rights, freely withdrawal of cooperation and explaining the questions and doubts on questions by the researchers. The questionnaires were completed by the subjects themselves. For those subjects that could not complete the questionnaire by themselves, it was filled by the researchers without any interferrence. In this research, content validity was used to determine the validity. Besides, using Cronbach's alpha the reliability was calculated to be 0.84 . In this research, descriptive and inferential statistics were used to analyze the data obtained from the subjects. Using definite methods, data was, summarized, classified, and presented in statistical Tables by software SPSS22. After data collection, according to the research objectives, the data were analyzed by statistical comparison of means, t-test and Pearson test. Cronbach's alpha of the patient 
rights questionnaire was analyzed based on the existing axes. The degree of reliability of the respect to patient privacy and secrecy principle (0.82), desirable health care (0.85), access to efficient compliants system (0.85), patient's access to appropriate and adequate information $(0.80)$ and freely choice and decision making $(0.88)$ in receiving the hospital sevices were calculated at a Cronbach's alpha of $88 \%$ with a mean of 0.84 . The status of observance of patient rights charter from nurses' view point was investigated by demographic variables. Due to the presence of three internal and surgical wards and a variety of patients and nurses, each ward was analyzed separately. The city of Gonabad already had two hospitals that has been integrated into a larger hospital since 2017 that is well equipped and provide the best health care services for about 1000000 population with the coverage of other cities $100 \mathrm{~km}$ far. There is also a helicopter for emergencies in south of Khorasan-e-Razavi province. This hospital is a comprehensive hospital that provides all needed services in the region. The referred patients to this hospital are diverse and from different cultures. Besides, the nurses are graduated from different universities and cities of the country usually with slightly different cultures.

\section{Results}

Based on the results of the present study, the following explanations are given in the Tables below.

Table 1 Frequency distribution of samples (patients and nurses) based on demographic information

\begin{tabular}{|c|c|c|c|c|c|}
\hline \multirow[t]{2}{*}{ Samples } & \multirow[b]{2}{*}{ Demographic Information } & \multicolumn{2}{|c|}{ Patients } & \multicolumn{2}{|c|}{ Nurses } \\
\hline & & Frequency & Percent & Frequency & Percent \\
\hline \multirow{2}{*}{ Gender } & Male & 30 & $30 \%$ & 40 & $40 \%$ \\
\hline & Female & 70 & $70 \%$ & 60 & $60 \%$ \\
\hline \multirow{2}{*}{ Marital status } & Single & 37 & $37 \%$ & 30 & $30 \%$ \\
\hline & Married & 63 & $63 \%$ & 70 & $70 \%$ \\
\hline \multirow{5}{*}{$\begin{array}{l}\text { Education } \\
\text { Degree }\end{array}$} & Prediploma & 65 & $45 \%$ & - & - \\
\hline & Diploma & 25 & $25 \%$ & - & - \\
\hline & Associate & 15 & $15 \%$ & - & - \\
\hline & Bachelor & 12 & $12 \%$ & 90 & $90 \%$ \\
\hline & Master\&Higher & 3 & $3 \%$ & 10 & $10 \%$ \\
\hline \multirow{5}{*}{$\begin{array}{l}\text { Job Experience } \\
\text { (Years) }\end{array}$} & $1-5$ & - & - & 30 & $30 \%$ \\
\hline & $6-10$ & - & - & 25 & $25 \%$ \\
\hline & $11-15$ & - & - & 20 & $20 \%$ \\
\hline & $16-20$ & - & - & 18 & $8 \%$ \\
\hline & 21-25\& higher & - & - & 7 & $7 \%$ \\
\hline
\end{tabular}

The Table 1 shows that among 100 patients who were participated in this study, $70 \%$ were female and $30 \%$ male and among the nurses $40 \%$ were male while $60 \%$ of them were female. The percentage of married individuals among nurses regarding their recruitment age compared with that of patients was $63 \%$ to $70 \%$.

Table 2 Patient awareness of Patient rights

\begin{tabular}{lccc}
\hline Patient right charter \& its Dimentions & Mean \pm SD & The score range & The score range possible \\
\hline Receiving desirable health services & $18.4 \pm 3.7$ & $5-25$ & $5-25$ \\
Access to Information & $44.3 \pm 8.2$ & $18-66$ & $14-70$ \\
Respect to freely choice and decision making & $16.4 \pm 4 / 1$ & $6-27$ & $6-30$ \\
Respect to the patient privacy and secrecy principle & $27.1 \pm 4.5$ & $9-38$ & $8-40$ \\
Access to efficient compliants system & $14.7 \pm 2.7$ & $5-23$ & $5-25$ \\
Total & $81.3 \pm 17.6$ & $60-68$ & $38-65$ \\
\hline
\end{tabular}


Table 3 The relationship between the awareness of the Patients rights with the dimensions of the patienst rights charter from the patients viewpoint

\begin{tabular}{lcc}
\hline Variable & Corelation (r) & Sig. level (p) \\
\hline Receiving desirable health services & $0.27^{* *}$ & 0.042 \\
Access to information & $0.32^{* *}$ & 0.039 \\
Respect to freely choice and decision making & $0.38^{* *}$ & 0.019 \\
Respect to the patient privacy and secrecy principle & $0.68^{* *}$ & 0.021 \\
Access to efficient compliants system & $0.53^{* *}$ & 0.034 \\
\hline
\end{tabular}

$* * \mathrm{p}<0.01$

As it is seen in Table 3, the Pearson test significance level is 0.042 , which is less than the error level $(0.05)$, so the zero assumption (non-relation) is rejected and the intensity of this correlation is 0.27 . Therefore, at $95 \%$ confidence level, there is a meaningful positive correlation between the awareness of patient rights and satisfaction with health services. Besides, there is a significant positive relationship between patients' awareness of the their rights and access to information from the perspective of patients, respect for freely coice and desicion making, patients' awareness of their rightsand respecting the patient's privacy, and between the awareness of patient rights and access to the compliants system from the patients' view.

Table 4 Level of observing the different dimensions of the patients rights charter from the perspective of the patients under study

\begin{tabular}{lccc}
\hline & \multicolumn{3}{c}{ Patients right observance quality level } \\
\cline { 2 - 4 } Patient right charter dimentions & Good & $\begin{array}{c}\text { Moderate } \\
\text { Freq. (Percent) }\end{array}$ & $\begin{array}{c}\text { Poor } \\
\text { Freq. (Percent) }\end{array}$ \\
\hline Receiving desirable health services & N(\%) & $46(46)$ & $8(8)$ \\
Access to Information & $18(46)$ & $73(73)$ & $9(9)$ \\
Respect to freely choice and decision making & $42(42)$ & $34(34)$ & $24(24)$ \\
Respect to the patient privacy and secrecy principle & $90(90)$ & $6(6)$ & $4(4)$ \\
Access to efficient compliants system & $7(7)$ & $83(83)$ & $10(10)$ \\
\hline
\end{tabular}

Based on the results of Table 4, the observation priority of patient rights from the patient viewpoint were; to respect the patient privacy and secrecy principle, receiving desirable services, access to an efficient complaints system, patients access to appropriate and adequate information and respect to freely choice and decision making, respectively.

Awareness and observance of patient rights is one of the most important rights of patients. Therefore, nurses are required to be aware of patient rights and oberve them. According to the results of this study, the most weighted average belonged to the phrases for the awareness and observance of the patient rights by nurses that showed the highest level $(3.78 \%)$ for the question on the infection transmission "To what extent the patient has the right to prevent the transmission of various infections to his body during the operation" and the least weighted average (3.25\%) was related to this questions "To what extent does the patients have the right to know what actions is being done for them and how much the patient have right to access their physician and other members of the treatment group after discharge or during treatment".

The relationship between the awareness levels of nurses working in the hospital about the patients rights by demographic variables.

Based on the results of the Anova test, which is shown in Table 5, the mean score of patient rights was not significantly in different age groups. However, there was a significant difference between the different years of job experience in the hospital. The degree of observance of the patient rights was differentin terms of patient satisfaction according to age, but there was no difference in terms of work experience. Table 5 shows that there was no significant difference in the level of observance 
of patient rights in terms of gender in both male and female groups.

Table 5 The relationship between demographic characteristics of nurses with patients' rights and satisfaction

\begin{tabular}{|c|c|c|c|c|c|}
\hline \multirow{2}{*}{$\begin{array}{l}\text { Demographic } \\
\text { Variables }\end{array}$} & \multirow{2}{*}{ Dimentions } & \multicolumn{2}{|c|}{ Patient right observance } & \multicolumn{2}{|c|}{ Patient satisfaction } \\
\hline & & Mean & SD & Mean & SD \\
\hline \multirow{3}{*}{ Age (Year) } & $25-35$ & 27.3 & 16.5 & 24.3 & 13 \\
\hline & $36-45$ & 26.2 & 16.3 & 27.9 & 13.5 \\
\hline & $46-55$ & 25.4 & 15.4 & 23.2 & 11.59 \\
\hline \multicolumn{2}{|c|}{ P-value* } & \multicolumn{2}{|c|}{0.46} & \multicolumn{2}{|c|}{0.036} \\
\hline \multirow{5}{*}{$\begin{array}{l}\text { Job Experience } \\
\text { (Year) }\end{array}$} & $1-5$ & 25.14 & 15.66 & 31.26 & 14.31 \\
\hline & $6-10$ & 26.36 & 16.3 & 22.14 & 15.36 \\
\hline & $11-15$ & 31.30 & 14.61 & 25.66 & 17.01 \\
\hline & $16-20$ & 27.14 & 15.33 & 28.78 & 16.14 \\
\hline & 20\&higher & 29.33 & 14.02 & 29.29 & 15.54 \\
\hline \multicolumn{2}{|c|}{ P-value* } & \multicolumn{2}{|c|}{0.042} & \multicolumn{2}{|c|}{0.156} \\
\hline \multirow{2}{*}{ Gender } & Man & 8.4 & 6.5 & 28.2 & 11.6 \\
\hline & Woman & 7.8 & 6.3 & 25.5 & 13.2 \\
\hline \multicolumn{2}{|c|}{ P-value** } & \multicolumn{2}{|c|}{0.15} & \multicolumn{2}{|c|}{0.91} \\
\hline \multirow{2}{*}{ Marital status } & Single & 7.6 & 6.5 & 26.7 & 12.4 \\
\hline & Married & 7.1 & 6.2 & 25.3 & 13.9 \\
\hline \multicolumn{2}{|c|}{ P-value* } & \multicolumn{2}{|c|}{0.7} & \multicolumn{2}{|c|}{0.24} \\
\hline \multirow{2}{*}{$\begin{array}{l}\text { Educational } \\
\text { Degree }\end{array}$} & Bachelor & 26.9 & 15.5 & 29.7 & 13.6 \\
\hline & Master & 26.3 & 12.4 & 28.6 & 11.4 \\
\hline \multicolumn{2}{|c|}{ P-value** } & \multicolumn{2}{|c|}{0.331} & \multicolumn{2}{|c|}{0.334} \\
\hline
\end{tabular}

\section{Discussion}

The results of this study showed that the patients' awareness of their rights is in a low status but patient rights observance from the patients' perspective in Bohlool hospital was at a good level. The results also showed that prioritizing of the patient rights observance from the patients' viewpoint in Bohlool hospital, were respectively,receiving desirable services and access to an effective complaints system, respecting the patient privacy, patient access to appropriate and sufficient information and the right to choice and make decision freely in receiving services. The results of this research are in accordance with those of Pishgar research [19], on the knowledge and observance of patient rights onoperating room personnel at Jahrom University of medical sciences hospitals, and those of Borhani research [20], on the awareness of the patient rights charter in Kerman city. Factors such as higher age, lower education, living in the village, shorter admission duration and hospitalization in the domestic and infectious wards were the influencing factors on the patient rights. Kolahi and Sohrabi reported that in average, $64 \%$ of the patient rights were observed mostly in the axis of familiarity with their physician name and non-discrimination in race, culture and religion in the health services delivery. In the current study the patients' satisfaction had in the highest level for the desirable health services [21].

Astereki et al. [22] stated that the average rate of patients' satisfaction was 3.45 and in the current study, this average was 3.68. In Voskooi study [5], the patients' satisfaction with respect to observing their rights was $53.2 \%$ and its lowest level blonged to the right of freely choice and decision making. Similarity, in the current study, the satisfaction degree in this axis was at the lowest level. Gahormanian et al. [23], found a strong and positive relationship between the days of admission and need for nursing 
interventions on the observance of their rights in patients' views. In the present study, the number of hospitalization days in hospital had a significant and negative correlation so that with the increase in the number of hospitalization days, the level of satisfaction was reduced. One of its factors can be being far from family and low welfare services. Basiri [11], stated that the rate of right observance was $69.1 \%$ and there was no relation between the increase of patient age and the number of admission days. However, in the current study the observance of patient rights was related with the increase in the patient age and the number of admission days. So, older patients had a better view on patient rights observation, while the level of satisfaction was reduced with the increase of admission days in hospital. Parsapur [24], reported that the observation of patient rights from their viewpoints was at the highest level for receiving desirable health services, and, access to adequate information, and, it was at the lowest level for choosing and deciding freely which was in line with the current study findings. The study by Kolroozi et al. [25] also confirmed these findings. To examine the relationship between demographic variables of nurses (age, gender, marital status, work experience and educational degree) with the observance of patient rights, Pearson correlation was used. The results showed that the level of significance was less than 0.05 which was less than error level (0.05). Therefore, the zero assumption (non-relation) is rejected, which indicates the relationship between nurses' demographic variables with the awarness and observance of patients' rights. The results of this research are consistante with those of Nekooi Moghadam [26], on patient's rights and observing it from patients' and nurses viewpoint, and Bokai [17] on the observation of patient rights by health care personnel from patients' viewpoints admitted to Shahid Sadoughi hospital in Yazd. The current study showed that the nurses were aware of the patient rights and these rights were observed from nurses' view in this hospital and the observance and awareness of the patient rights were at a good level. The findings of this study showed that nurses had a good knowledge about patient rights. Hooshmand [7] also found that $95.5 \%$ of nurses working in educational hospitals in Tehran had a high degree of awareness about the patient rights charter. Parsapour et al. reported that the awareness level of patient rights in Spain was $84 \%$ [18].

Asteraki et al. [22] reported that the knowledge level of health care personnel of Isfahan about the patient rights was excellent. These results indicate that nurses' awareness of the patient rights charter is moderate or above. This could be due to holding educational classes, implementing clinical governance policies in Iranian hospitalsand high participation of nurses in the implementation of these programs.

According to the findings of this research, the following suggestions are presented: To provide, strengthen, support and keep the patient rights through appropriate and wise management, To develop some plans for culture promotion instead of short and ineffective actions, To Provide necessary educations to patients through media, family and educational institutions, by making educational pamphlets about patient rights, inviting experts in this field and producing educational films about these subjects and to provide accurate and in time information about the disease, its stages and duration of treatment in all aspects of the individual health, medical care and other aspects of life to inform the patient about the condition in which he/she is and then establishment of a Quality Management Committee for hospitals wards with specific job descriptions and some plans to implement the quality assurance programs.

\section{Conclusion}

It was found that failure to observe the patient rights charter can endanger the health, safety and security of patients, as well as undermine the relationship between health care staff and patients, which ultimately leads to a reduction in the effectiveness 
of patient care. It is obvious that patients' awareness, their participation in decision making and respect for their rights can speed up their recovery, it reduces the hospitalization time and makes them participate actively in their treatment. Besides, the annoncement of the patient rights charter jast as a formal written letter, without any follow up, can not change the hospitalservices. Therefore, some mechanisms should be performed to control and train physicians, nurses and even other staffs. Patients' rights formulation is a critical step towards balancing the cost and access to care system and within the process of ousourcing the health services it plays an important role to build public trust. In fact, the development of patient rights involves a series of methods and policies that are implemented in a coordinated manner in different parts of a center to achieve therapeutic goals, and determine the scope of caring and what are allowed to do or not. The present study, which is in the field of behavioral sciences studies, had some limitions such as: the difficulty of facing acute patients and observing certain principles, the precaution in generalizing the results widely to other statistical populations, unability to declare the results in a causal way and the probability of bias in filling the questionnaires by the participants. Therefore, in order to solve these challenges or minimize them by authorities, the following suggestions are presented.

\section{Acknowledgements}

The authors would like to thank the research authorities in Islamic Azad and Gonabad Medical Universities, Bohlool hospital of Gonabad city for their contribution and all the patients and nurses who participated in the study.

\section{Authors' contributions}

Study design: FB, ARA

Data collection and analysis: ARA, BAA, ARA Manyscript preparation: FB, ARA

All authors have read and approved the final version

\section{Conflict of Interest}

"The authors declare that they have no competing interests".

\section{Funding}

The author (s) received no financial support for the research, authorship and/or publication of this article.

\section{Availability of data and materials}

The datasets used and/or analyzed during this study are available from the corresponding author on reasonable request.

\section{References}

1- Davati A, Seidmortaz SS, Azimi A, Saeed AS. A study on knowledge of general practitioners on the charter of patients' rights. Daneshvar Medicine2011; 18(91): 81-8.

2- Sarbaz M, Kimiafar Kh. Comparative study of patients' rights in some developed country and presenting a model for Iran. Health Information Management2011; 8(2): 218-27.

3- Almoajel Alyah M. Hospitalized patients' awareness of their rights in Saudi governmental hospital. Middle East J Sci Res2012; 11(3): 329-335.

4- Yousuf RM, Fauzi ARM. Shah A. Hospitalized patients' awareness of their rights: a cross-sectional survey from a tertiary care hospital on the east coast of Malasiaya. Singapore Med J2009; 50(5): 494-9.

5- Vaskooei Eshkevari K, Karimi M, Asnaashari H, Kohan N. The assessment of observing patients' right in Tehran University of Medical Sciences' hospitals. Iranian Journal of Medical Ethics and History of Medicine2009; 2 (4):47-54.

6- Aydemir I, Ongoren B. Patient rights practice in turkey. Academic Research International2013; 4(2): 520-9.

7- Hooshmand A, Joolaee S, Mehrdad N, Bahrani N. Nurses' information and their view points about patient's rights and practical facilitators in clinics. Hayat2007; 12(4): 57-66.

8- Rider M E, Makela C. A comparative analysis of patients' rights: an international perspective. Int $J$ Consum Stud2003; 27(4): 302-12.

9- Arab M, Zarei A. Awareness of the managers of state hospitals in Tehran city from patients' rights. Journal of Health Administration2008; 10(12): 7-27.

10- Soheili M, Peiman A, Tabarsi B. A comparative study on midwives 'and mothers' opinion of adherence to patient's rights. Tehran: The 1st Congress on Islamic Sciences \& human rights, 2018. COHE01_038. 
11- Basiri Moghaddam K, Basiri Moghadam M, Moslem A, Ajam Zibad H, Jamal F. Awareness of patients and staffs of medical group of the Patients' rights charter and its compliance in one of the educational hospitals in Gonabad. Ofough-e-Danesh2011; 17(1): 45-54.

12- Khodamoradi KAS, Jalali S, Mobini N. Assessing undergraduate and graduate nursing students 'knowledge of patients' rights. Medical Ethics Journal2010; 4(12): 133- 48 .

13- Larijani B, Zahedi F. Medicine and modern medical ethics. Iranian Journal of Diabetes and Metabolism2005; 4(0): 1-11.

14- Vivian LM, Naidu CS, Keikelame MJ, Irlam J. Medical students' experiences of professional lapses and patients' rights abuses in a South African health sciences faculty. Acad Med2011; 86(10):1282-7.

15- Shiraz B, Shamim MS, Shamim MS, Ahmed A. Medical ethics in surgical wards: knowledge, attitude and practice of surgical team members in Karachi. Indian $J$ Med Ethics2005; 2(3): 94-96.

16- Weaver R, Wilson I. Australian medical students' perceptions of professionalism and ethics in medical television programs. BMC Med Educ2011; 11(1): 50.

17- Bokaei M, Anjazab B, Fotouhi Z, Sorouri M, Faraj Khoda T, Abbasi M. Examination of patients' knowledge of the patients' rights in Shahid Sadoughi hospital in Yazd at 2010. Medical Ethics2011; 5(17): 11-26.

18- Parsapoor A, Mohammad K, Malek Afzali H, Ala'eddini F, Larijani B. Necessity of observing patient's rights: a survey on the attitudes of patients, nurses and physicians. J Med Ethics Hist Med2012; 5(2): PMID: 23908755, PMCID: PMC3713703.

19- Pishgar Z, Parnean R, Rahmanean S, Shadfard Z.
Recognizing and respect for patients' rights by operating room personnel of hospitals affiliated with Jahrom University of Medical Sciences. Education \& Ethics In Nursing2015; 4(3): 49-56.

20- Borhani F, Abbaszadeh A, Nakhaee N, Roshanzadeh $\mathrm{M}$. The relationship between moral distress, professional stress, and intent to stay in the nursing profession. $\mathrm{J} \mathrm{Med}$ Ethics Hist Med2014; 7(3): 2-8.

21- Kolahi AA, Sohrabi MR. Surveying the knowledge of patients referring to Imam Hossein Hospital from patients' rights charter in 2002. Teb-e-Tazkiyeh2008; 16(3-4): 65-76.

22- Astaraki P, Mahmodi G, Anbari K, Hosseini N. Evaluation of respect for patients' rights from the viewpoint of hospitalized patinents in Shohada Ashayer hospital of Khoramabad city in 2013-2014. Yafteh2015; 17(1): 5-14.

23- Ghahremanian A, Rahmani A, Zamanzade V, Mohajjelagdam AR. Respecting patients' privacy during nursing care in the viewpoint of Patients and nurses. Iran Journal of Nursing2008; 21(55): 37-45.

24- Parsapoor A, Bagheri A, Larijani B. Patients' rights charter in Iran. Acta Med Iran2014; 52(1): 24-8.

25- Kolrouzi F, Dadgar F, Zareian A. Satisfaction of patients from the performance of health care teams in performancing the provisions of the charter of patients' rights. Journal of Military Medicine2010; 12(3): 143-8. 26- Nekouei Moghaddam M, Amir Esmaeili MR, Ghorbaninia R, Sharifi T, Tabataba'I SS. Knowledge of patients' rights and their observance from the perspective of patients and nurses: a study in limited surgical centers in Kerman province in 2013. Bioethics Journal2014; 4(11): 31-56.

Copyright $\subseteq 2016$ ASP Ins. This open-access article is published under the terms of the Creative Commons Attribution-NonCommercial 4.0 International License which permits Share (copy and redistribute the material in any medium or format) and Adapt (remix, transform, and build upon the material) under the Attribution-NonCommercial terms. 